International Journal of Stem Cell Research and Transplantation (1JST)

ISSN: 2328-3548

\title{
Ultrastructural Characterization of a Developing Pericytic Microtumor in the White Matter Post Laceration
}

Pais $\mathrm{V}^{1 *}$, Danaila $\mathrm{L}^{1}$, Pais $\mathrm{E}^{2}$

Research Article

\begin{abstract}
${ }^{1 *}$ Department of Neurosurgery, National Institute of Neurology and Neurovascular Diseases, 10-12 Soseaua Berceni, Sector 4, Postal Code 41914, Bucharest, Romania

${ }^{2}$ Spectral Molecular Imaging, Los Angeles, California, United States
\end{abstract}

Abstract
We studied by transmission electron microscopy an intraparenchymatous mass within white matter found in a patient operated on
for cerebral laceration. According to the ultrastructural criteria, this mass was diagnosed as an incipient hemangio-pericytoma, being
composed by staghorn vasculature and tumoral cells with appearance of pericytes, more or less phenotipycally changed. The prolif-
erating cells were almost nondifferentiated as well as similar to pericytes and vascular smooth muscle cells, i.e., heterogenous in their
phenotype and "regenerative" in the ultrastructural features. Precise origin and fate of these metabolically active cells is not known.
Surprisingly, the microtumor was delineated by numerous reticulin fibers and by long prolongations of cerebral cordocytes, which may
have a protective role against tumor growth in the brain parenchyma. However, this is the first ultrastructural study which has to be
continued for such unusual cases in the brain.
Keywords: Bain Laceration; Tumorigenesis; Intraparenchymatous Hemangiopericytoma; Pericytes/Perivascular Cells; Stem Cells;
Ultrastructure.

\section{*Corresponding Author:}

Viorel Pais,

Collaborator, Department of Neurosurgery,

National Institute of Neurology and Neurovascular Diseases,

Bucharest, Romania

Tel: (+40)31-409-7790 ; Fax: (40)21-334-6463;

E-mail: viorelpais@hotmail.com

Received: January 09, 2013

Accepted: March 01, 2013

Published: March 13, 2013

Citation: Pais V, Danaila L, Pais E. (2013). Ultrastructural Characterization of a Developing Pericytic Microtumor in the White Matter Post Laceration, Int J Stem Cell Res Transplant, 01(01), 01-07. doi: http:/ / dx.doi.org/10.19070/2328-3548-130001

Copyright: Pais $\mathbf{V}^{\odot}$ 2013. This is an open-access article distributed under the terms of the Creative Commons Attribution License, which permits unrestricted use, distribution and reproduction in any medium, provided the original author and source are credited.

\section{Introduction}

Renewed interest in the role of developmental pathways in tumorigenesis has led to more recent data on putative cancer stem cell population in brain as well as in other organs. The fate of proliferating and differentiatingpericyte-like cells in the microvessels of brain parenchyma derived most probable from mesenchymal stem cells or adult pericytes and should not be ignored because these cells may undergo malignant transformation to a rare, but aggresive tumor: hemagiopericytoma. Primary central nervous system (CNS) hemangiopericytoma is a predominantly meningeal based and traditionally thought to arise from spindle cells (pericytes) in the vicinity of the blood vessels. An intraparenchymal tumor mass is little known in literature [1,2,3], but not described ultrastructurally. This is the first study concerning ultrastructural description of a tumoral mass into white matter post laceration with pericytic abnormal proliferation.

\section{Materials and Methods}

We examined by transmission electron microscopy samples of brain tissue in a clinical case (male, 61-yearold) operated on for brain laceration following a severe cranial trauma involving the deeper white matter. Multiple blocks were processed using standard procedures for conventional electron microscopy. The specimens were fixed in a solution of $2.5 \%$ buffered glutaraldehyde to a $\mathrm{pH}$ of $7.2-7.3$ for 4 hours. They were later postfixed in a solution of $1 \%$ buffered osmium tetroxide for 1 hour, dehydrated with increasing concentrations of alchohols in water, and embedded in resin epoxy (Epon 812). The resin was polymerized to $700 \mathrm{C}$ for 72 hours. Multiple ultrathin sections, $70 \mathrm{~nm}$ thick, were cut with an ultramicrotome and mounted on specimen grids covered with plastic films. These sections were contrasted with $2 \%$ uranyl acetate solution as well as Reynolds lead citrate solution. The specimens were then examined under a JEM $1200 \mathrm{EX}$ (JEOL) transmission electron microscope. The electron photomicrographs were processed on a computer and converted into images.

\section{Results and Discussions}

We examined ultrastructurally an abnormal micronodule of polygonal and spindle cells with features of pericytes and exhibiting staghorn vasculature, like in hemangio-pericytoma, within the white matter in a 61-year-old male, operated on for laceration of the brain. This microtumor was hypercellular and hypervascular in the white matter which was degenerated,with numerous immune cells, some of them infiltrated in the tumoral mass which contained metabolically active cells. Endothelial cells were polygonal or elongated, having a nucleus with incisures and an abundant cytoplasm, rich in poliribosomes, mitochondria, and rough endoplasmic reticulum (RER). A centriole was visible in some cells 
suggesting their mitotic potential. Cell membrane was irregular, undulated on the abluminal side and with many short microvilli, some detached in the lumen by apical constrictions. Adjacent to the endothelial cells, overlapping spindle cells rich in filaments were distributed mainly to the cell periphery as contractile microfilament bundles and a large number of plasmmalemal vesicles. Some pericyte-like cells contained large glycogen deposits. Extracellular space between endothelial cells and adjacent cells were filled with an abundant collagenous matrix suggesting an intense synthesis of collagen type IV and other proteins within RER cisterns, very numerous in these cells (Figs.1a, b, c). It is remarkable that the endothelial cells showed cell membrane folding (Fig.2). Numerous cells proliferated around the vessels were characterized by a voluminous nucleus and a little cytoplasm or, conversely, with many irregular prolongations, between which it was seen a significant number of immune cells infiltrated in microtumor mass and present within the adjacent white matter, especially macrophages and neutrophils (Fig.3). Most of the tumoral cells exhibited a voluminous and euchromatinized nucleus and a prominent nucleolus with a little cytoplasm containing poliribosomes, mitochondria, and rough endoplasmic reticulum. Some tumoral cells, with abundant cytoplasm showed proteolysis areas. Around the cells there were thin fibrils, more abundant to the micronodule periphery. Interestingly, a thin basement membrane surrounded this tumoral formation (Fig.4). Thin connective fibrils are distributed throughout microtumor, surrounding each tumoral cell, and not the immune cells infiltrated in the tumoral mass (Fig.5). More interestingly it was the presence of long and thin prolongations of the interstitial cells from the white matter, so-called cordocytes, around this microtumor, while the myelinated axons were degenerated (Fig.6). Cordocytic processes were interconnected and overlapped,surrounding a large area of the tumoral micronodule,suggesting a barrier against developing tumor (Fig.7).However, in some places, these protective cell-barriers are missing (Fig.8), whereas in other zones, these thin cytoplasmic processes seem to be detached from tumoral micronodule and then degenerated. In those places there are numerous peripheral thin connective fibrils (Fig.9). Around the microtumoral mass, the white matter was degenerated, axons being charicatural in aspect, some glial cells, for example, oligodendrocytes, were seen in apoptotic stages (Fig.10), and numerous microglial cells loaded with autophagosomes, secondary lysosomes and vacuolar cyto-

Figure 1a. Intratumoral small capillary with characteristic aspect of staghorn, like in well developed hemangiopericytoma. Endothelial cells and surrounding pericytes have an abundant cytoplasm with numerous organelles suggesting their active metabolic state. $\mathbf{x} 800$

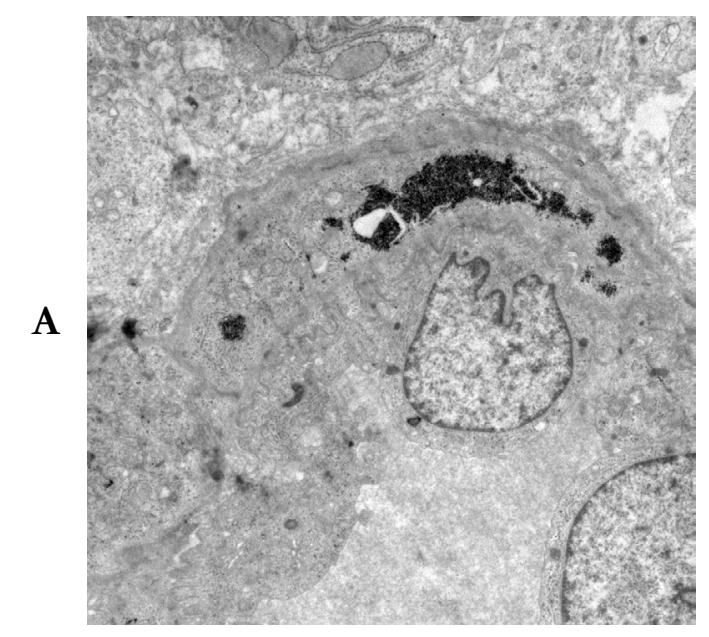

Figure 1b. Detail from Fig.1a showing particularly thick basement membranes, undulated endothelial cell membrane and cisterns of rough endoplasmic reticulum in the abnormal proliferated cells around the vessels. $\mathrm{x} 12,000$

Figure 1c. Endothelial cells of the capillary illustrated above displaying voluminous nuclei, abundant cytoplasm, irregular microvilli into lumen, and glycogen deposits in the surrounding cells. x10,000
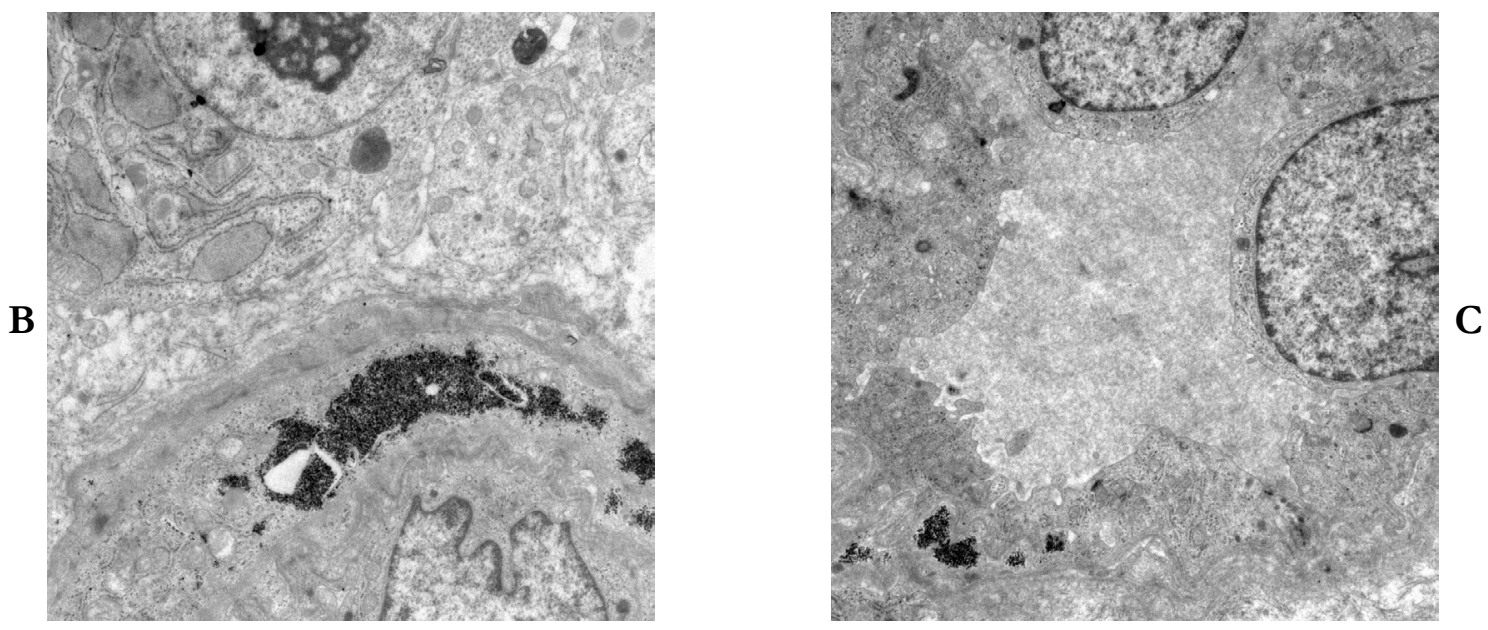
Figure 2. An endothelial cell with abundant cytoplasm, Golgi complex and cell membrane very undulated, and two pericytes with Golgi complex, miofilaments and plasmmalemal vesicles. x20,000

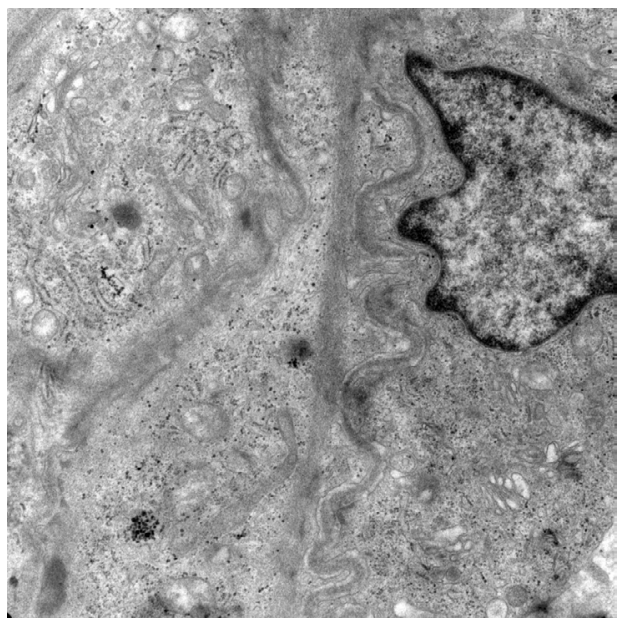

Figure 3. Tumoral cells with variable morphology around the capillary showing either voluminous nucleus and a little cytoplasm or irregular prolongations,suggesting their phenotypic changes,intermingled with infiltrated immune cells. $\mathrm{x} 10,000$

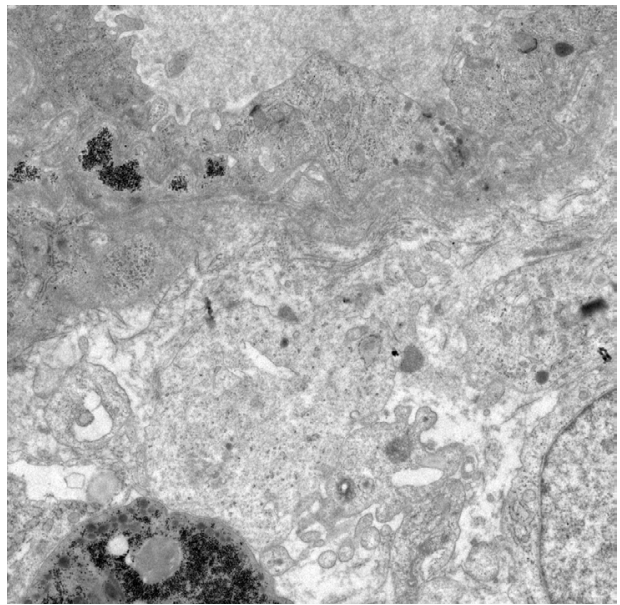

Figure 4. Tumoral cells having a voluminous euchromatic nucleus with some areas of genic activation, prominent nucleolus, a little cytoplasm, rich in poliribosomes, mitochondria and RER, surrounded by cells with several lytic cytoplasmic areas and immune cells as well, separated by reticulin fibers. Significantly, around the tumoral micronodule, there is a thin and continuous basal lamina. $x 12,000$

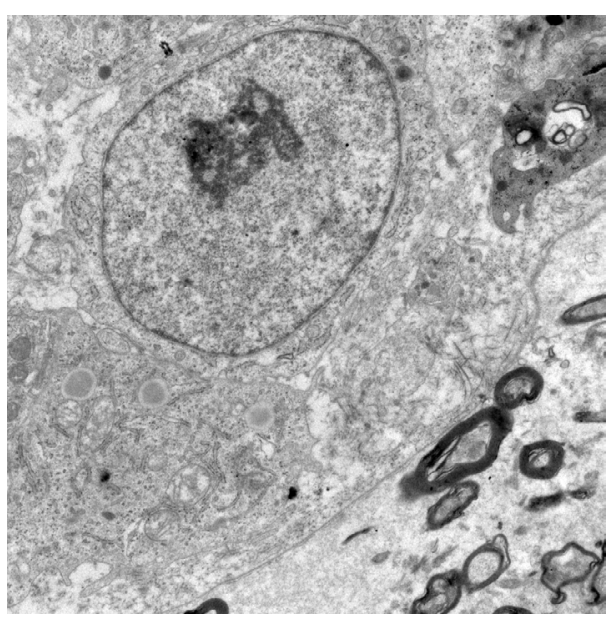

plasmic areas suggested autophagy (Fig.11). No isolated tumoral cells were seen in the adjacent white matter.

Herein, we analyzed by transmission electron microscopy a micronodule thought to be tumoral in nature, located within the white matter in a male patient who suffered a brain laceration. We observed an increased density of cells similar to each other, seemed to be derived from pericytes/perivascular cells which were tightly associated with modified endothelial cells of staghorn shaped vessels. These proliferated polygonal cells, surrounding endothe- lial cells in the so-called staghorn pattern are characteristic for hemangiopericytoma, and subsequently may develop into genuine intraparenchymatous tumor. It is possible that trauma has been involved, as a possible cause of that malignant cell deviation, because this factor is considered one of the causes of CNS sarcomas [4]. However, the ultrastructural findings were consistent with hemangio- pericytoma in early stage of microtumor. Recently it was reported an hemangio-pericytoma in the trigone of the lateral ventricle[5]. Hemangiopericytomas occur anywhere capillaries can 
Figure 5. Tumor cells with variable ultrastructure, separated by thin connective fibers, and neutrophils and macrophages infiltrated in the tumoral mass. $x 12,000$

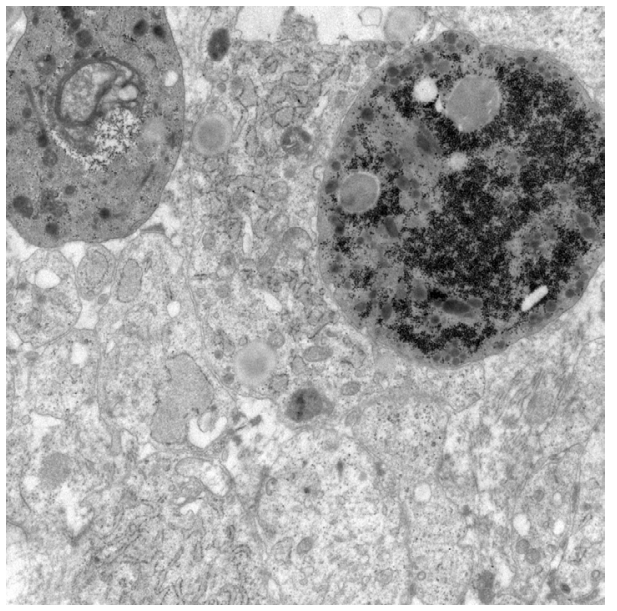

Figure 6. This image shows long cordocytic prolongations around the tumoral micronodule suggesting a physical barrier for tumor growth, and myelinating axons which are degenerated within the white matter. $\mathrm{x} 12,000$

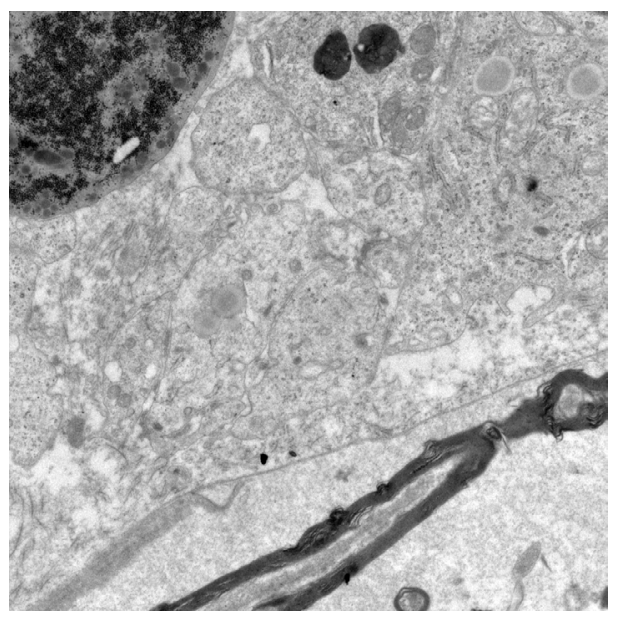

Figure 7: Cellular prolongations of the reactive cordocytes are irregular,interconnected, overlapped, and surrounded a large area of microtumor. $x 10,000$

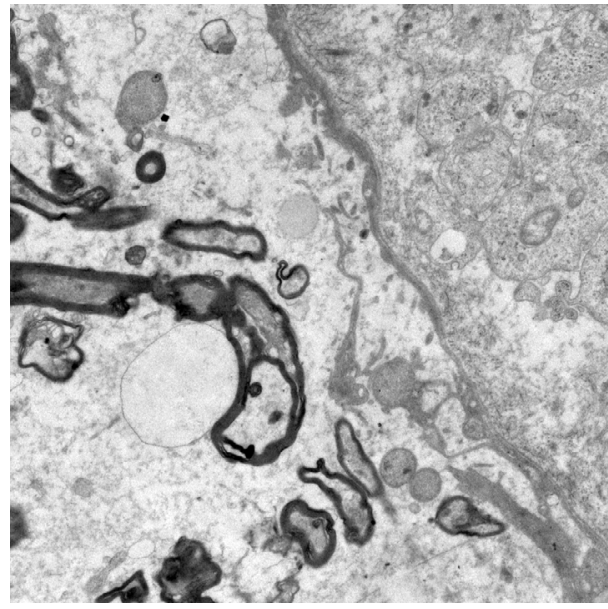

be found [6]. Histopathological features of a hemangio-pericytoma include sheets of homogeneous cells with uniform hypercelularity, abundant cytoplasm, oval nuclei with mild nuclear atypia, staghorn vascular pattern lined by flat endothelial cells which tend to bulge into the vascular lumen, whereas immunohisto-chemical results indicate pericellular reticulin, type IV collagen, pericellular basement membrane, CD34, MIC2, BCL2, EMA, factor XIIIa, Leu 7, CD99, vimentin, p53, variable smooth muscle actin, cytokeratin, desmin, claudin $[7,8]$. Pericytes show biochemical and physiological features similar to vascular smooth muscle cells, usually containing smooth muscle actin fibers and rich endoplas- mic reticulum. In our case, the cells adjacent to the endothelium were rich in filaments and the cells at distance were rich in RER. The tumoral cells were detached from the basal lamina encasement, but the entire tumoral micronodule was surrounded by a thin basal lamina which further was enwrapped by a long cordocytic process. Most importantly, these proliferation cells did not show signs of leaving the micronodule. Human pericytes express Robo1 and Robo4 receptors which in the presence of their ligand Slit2, spontaneously and PDGF-induced migration of pericytes is impaired [9]. Some studies have demonstrated altered pericyte morphology in response to traumatic brain injury and suggested 
Figure 8; Cordocytic processes are missing in some zones of microtumor, probable due to the degenerative processes during laceration. $\mathrm{x} 6,000$

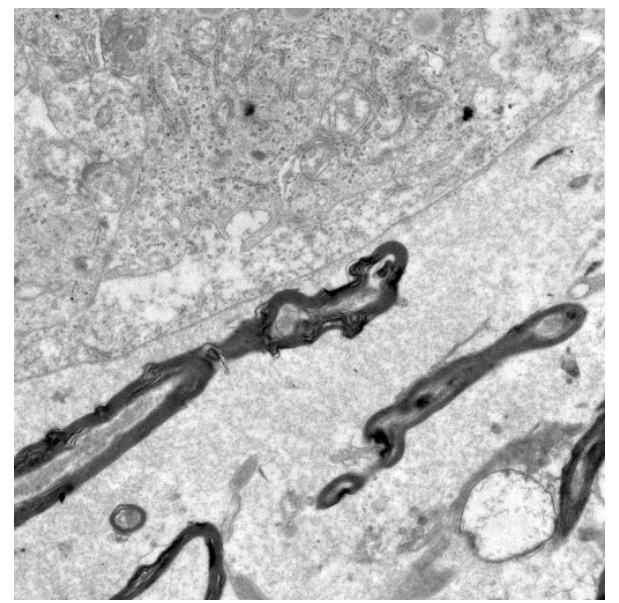

Figure 9. In some areas of the tumoral micronodule, cordocytic processes are detached from the periphery of microtumor and degenerated. Instead, there are numerous thin connective fibers. $\mathbf{x} 25,000$

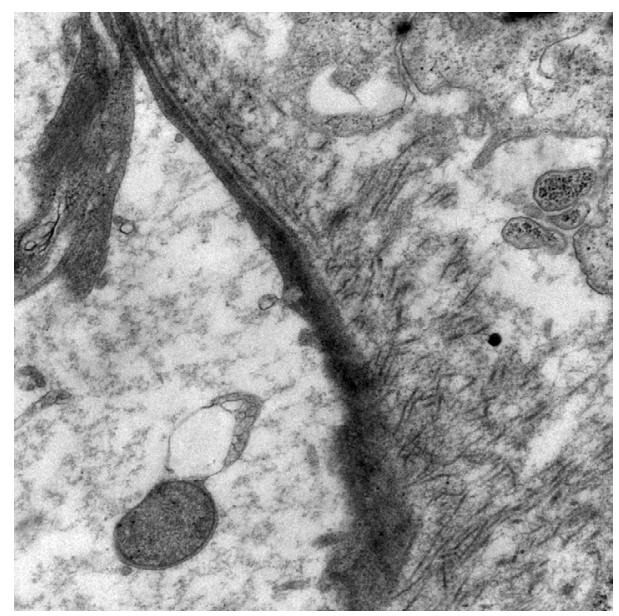

Figure 10: An oligodendrocyte from the degenerative white matter showing cytoplasm condensation and chromatin margination suggesting apoptosis. $\mathrm{x} 20,000$

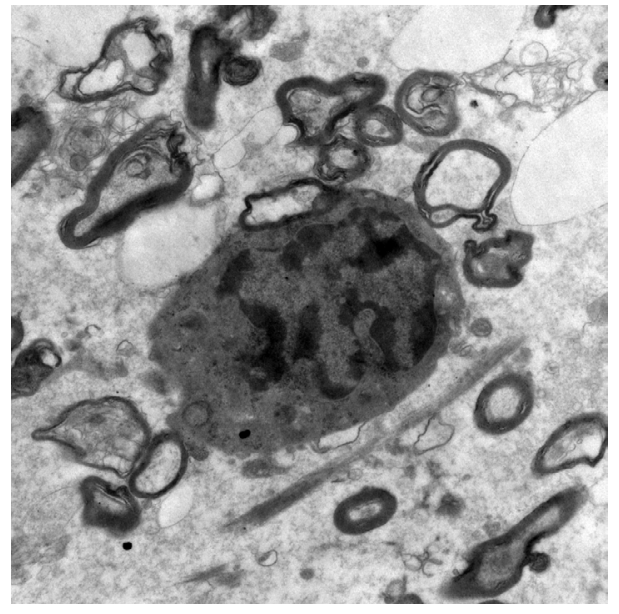

that they may leave the vessel wall [10]. Interestingly, in our case these cells have deposited to the micronodule periphery an abundant ECM including thin reticulin fibers and amorphous collagen. Most importantly it was the presence of cordocytes around the nodule, despite all the cells in neuropil and microvessels were degenerated due to the posttraumatic necrosis. Even excessive intracellular deposits of glycogen deposited in the vessel wall may activate the complement and cause necrosis. Tissue necrosis triggers an inflammatory reaction that clears the wound from dead cells and matrix debris. Clearance of apoptotic neutrophils, recruitment of inhibitory monocyte subsets and regulatory $\mathrm{T}$ cells, macrophage differentiation and pericyte-endothelial interaction may play an active role in restraining post laceration inflammation. Multiple molecular signals may be involved in suppresing the inflammatory cascade, downmodulation of cytokine responses and other biochemical events. Interestingly, in the microtumoral mass there were infiltrated many neutrophils as well as macrophages.

In the context of hypoxia, monocytes migrate into tissue where they differentiate into macrophages, cells that secrete several molecules important for activation of angiogenesis, such as granulocyte-colony stimulating factor, PDGF, TGF- $\alpha$, FGF-2 and IGF-1. 
Figure 11 A microglial cell from the white matter displaying many lysosomal structures and cytoplasmic areas of proteolysis whereas nucleus is normal, suggesting autophagy. $\mathrm{x} 20,000$

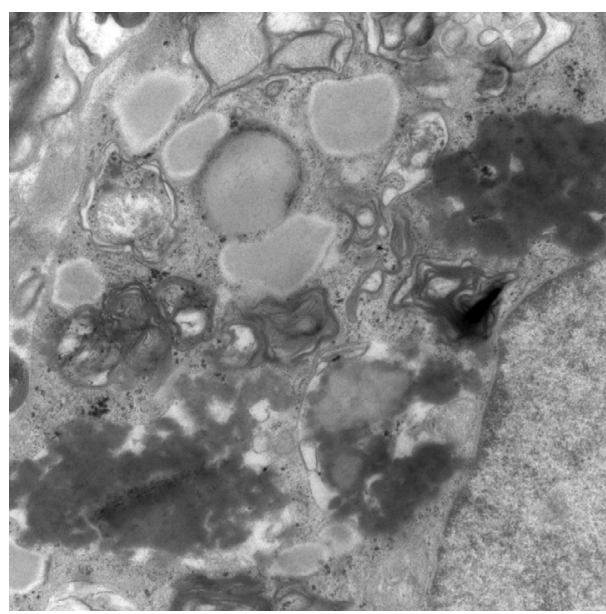

In tumor angiogenesis as in other situations, pericytes and their maturation should not be ignored [11,12] Microvascular pericytes/perivascular cells throughout CNS have neural stem/progenitor cells activity in response to ischemia and can generate neurons having an important role in CNS restoration following brain injury, such as stroke [13], but also these cells may undergo malignant transformation following brain injury, such as trauma. However, the origin, identity and subtypes of these microvascular pericytes/perivascular cells remain to be determined. During their differentiation in the vascular wall, pericytes can be fibroblastoid in phenotype, with an extensive RER or similar to the smooth muscle cells containing numerous contractile microfilaments as in the case herein illustrated. On the other hand, from the precursor/stem cells or even mature perivascular pericytes may arise cells with neural phenotype or even epithelial phenotype, when the cells are joined by zonula adherens or desmosomes well-developed. In respect to the cordocytes, these cells are always found outside the perivascular pericytes, either normal or abnormal in phenotype, like in this case. Characteristic morphology and ultrastructure of hemangiopericytoma are now well defined in meninges and other organs $[14,15,16]$. The protective role of cordocytes as a barrier against microtumor development remain to be confirmed $[17,18,19]$. During different cell processes either in normal or abnormal conditions, different cellular types may undergo a form of programmed cell death, mainly apoptosis and autophagic apoptosis or autoschizis, as seen in the case here illustrated, affecting especially neutrophils, macrophages/microglial cells, oligodendrocytes, astrocytes, but not tumoral cells, very active metabolically, proving their immortal phenotype at the onset of tumorigenesis [20,21]. However, in this stage of tumor growth, the tumoral cells are not seen disseminated into adjacent brain parenchyma, and the role of the stem cells which actually orchestrate cell growth and proliferation within tumor remain to be determined [22,23].

In conclusion, this ultrastructural study reveals the apparition of a hypercellular and hyper-vascular mass, with very active metabolic endothelial and pericytic cells, within the degenerated white matter due to the cerebral laceration. This intraparenchymatous mass is considered a microtumor belonging to the hemangiopericytoma according to the histopathologic features (cells similar to the perivascular pericytes and vessels with staghorn model). Despite its small size, this microtumor showed a large variability of cell ultrastructure, from nondifferentiated cells to cells similar with pericytes, and even vascular smooth muscle cells, either containing a voluminous nucleus with prominent nucleolus and only a rim of cytoplasm or, respectively, an abundant cytoplasm with dilated RER cisterns and bundles of myofilaments. Surprisingly, around the microtumor we found a thick basement membrane and long cordocytic processes as evidence of the role of these protective cells in the immune surveillance. However, the precise origin and fate of these cells with genuine tumoral characteristics of hemangiopericytoma remain impredictible. Therefore, further studies are necessary to evaluate ultrastructurally the behavior of pericytes and their precursor/stem cells in the wall of human cerebral microvessels during tumorigenesis.

\section{References}

[1]. Shetty PM, Moiyadi AV, Sridhar E (2010) Primary CNS Hemangiopericytoma presenting as an intraparenchymal mass--case report and review of literature. Clin Neurol Neurosurg 112(3): 261-264.

[2]. Kuzeyli K, Cakir E, Karaarslan G, Usul H, Reis A, et al.(2003) Primary parenchymal cerebral cystic haemangiopericytoma: a 5-year follow up of disease progression.Australas Radiol 47(1): 88-91.

[3]. McLendon RE, Enterline DS, Tien RD, Thorstad WL,Bruner JM (1998) Tumors of central neuroepithelial origin. In: Russell \& Rubinstein's Pathology of Tumors of the Nervous System. (2ndvol), Oxford University Press, Inc., New York.

[4]. Haddad GF, Al-Mefty O (1995) Meningeal sarcoma. In: Kaye AH Jr, Laws ER,editors, Brain Tumors: An Encyclopedic Approach.Churchill Livingstone, New York.

[5]. Tanaka T, Kato N, Arai T, Hasegawa Y, Abe T (2011)Hemangiopericytoma in the trigone of the lateral ventricle. Neurol Med Chir (Tokyo)51(5): 378-382.

[6]. Lutzen U, Engellandt K (2006) Late distant metastases of intracranial hemangio pericytomas. Rontgenpraxis. 56(3): 93-97.

[7]. Purandare NC, Dua SG, Rekhi B, Shah S, Sharma AR, etal. (2010) Metastatic recurrence of an intracranial hemangiopericytoma 8years after treatment: report of a case with emphasis on the role of PET/CT in followup. Cancer Imaging 10: 117-20.

[8]. Al-Brahim N, Devilliers R, Provias J (2004) Intraventricular hemangiopericytoma. Ann Diagn Pathol 8(6): 347-351.

[9]. Guijarro-Munoz I, Cuesta AM, Alvarez-Cienfuegos A,Geng JG, Alvarez-Vallina L, etal. (2012) The axonal repellent Slit2 inhibits pericyte migration:potential implications in angiogenesis. Exp Cell Res318(4): 371-378.

[10]. Dore-Duffy P, Owen C, Balabanov R, Murphy S, BeaumontT, et al. (2000) Pericyte migration from the vascular wall in response to traumatic brain injury. Microvasc Res 60(1): 55-69.

[11]. Collet GD, Canfield AE (2005) Angiogenesis and pericytes in the initiation of ectopic calcification. Circ Res 96(9): 930-938.

[12]. Fakhrejahani E, Toi M (2012) Tumor angiogenesis: pericytes and maturation are not to be ignored. J Oncol 2012: 261750.

[13]. Nakagomi T, Molnar Z, Nakano-Doi A, Taguchi A,Saino O, et al. (2011) Ischemia- induced neural stem/progenitor cells in the pia mater following cortical infarction.Stem Cells Dev 20(12): 2037-2051.

[14]. Arseni C, Pais V (1987). Ultrastructure of intracranial tumours and peritumoral zones. In: Romanian Neurosurgery. (4thvol), Romanian Academy Publishing House, Bucharest.

[15]. Pais V (1983) Ultrastructura pielii umane. Editura Medicala, Bucuresti.

[16]. Danaila L, Arsene D, Carp N (2005) Morfopatologia siclinica procese- 
lor expansive ale sistemului nervos central. Editura Academiei Romane, Bucuresti.

[17]. Pais V, Danaila L (2008) The interstitial cells of the human brain. An atlas of light and electron microscopy. (2ndedn), Cartea Universitara, Bucharest.

[18]. .Pais V, Danaila L (2010) The interstitial cells of the human brain. An atlas of light and electron microscopy. (3rdedn), Ars Academica, Bucharest.

[19]. Pais V, Danaila L, Pais E (2012) From pluripotent stem cells to multifunctional cordocytic phenotypes in the human brain: an ultrastructural study. Ultrastructural Pathology 36(4): 252-259.

[20]. Pais V, Danaila L, Pais E (2012) A comparative ultrastructural study of a new type of autoschizis versus a survival cellular mechanism that involves cell membranes of cerebral arteries in humans.Ultrastructural Pathology 36(3): 166-170.

[21]. Pais V, Danaila L (2011) The ultrastructure of the dying cells in the brain. An atlas of transmission electron microscopy. Ars Academica, Bucharest.

[22]. Dirks PB (2006) Cancer: stem cells and brain tumours. Nature 444(7120): 687-688.

[23]. Visvader JE, Lindeman GJ (2012) Cancer stem cells:current status and evolving complexities. Cell Stem Cell 10(6): 717-728. 